\title{
Godelian Ontological Arguments
}

\section{GRAHAM OPPY}

There is now a considerable secondary literature on Godel's ontological arguments; in particular, interested readers should consult Sobel (1987), Anderson (1990) and Adams (1995). In this note, I wish to draw attention to an objection to these arguments which has hitherto gone unnoticed. This objection does not depend upon fine details of the formulation of the arguments; I arbitrarily choose to develop the objection in connection with the formulation provided by Anderson.

In brief, the argument I shall consider may be summarised thus:

Definition 1: $\mathrm{x}$ is God-like iff $\mathrm{x}$ has as essential properties those and only those properties which are positive.

Definition 2: $\mathrm{A}$ is an essence of $\mathrm{x}$ iff for every property $\mathrm{B}, \mathrm{x}$ has $\mathrm{B}$ necessarily iff $\mathrm{A}$ entails B

Definition 3: $\mathrm{x}$ necessarily exists iff every essence of $\mathrm{x}$ is necessarily exemplified.

Axiom 1: If a property is positive, then its negation is not positive.

Axiom 2: Any property entailed by [= strictly implied by] a positive property is positive.

Axiom 3: The property of being God-like is positive.

Axiom 4: If a property is positive, then it is necessarily positive.

Axiom 5: Necessary existence is positive.

Theorem 1: If a property is positive, then it is consistent [=possibly exemplified].

Corollary 1: The property of being God-like is consistent.

Theorem 2: If something is God-like, then the property of being God-like is an essence of that thing.

Theorem 3: Necessarily, the property of being God-like is exemplified.

Given a sufficiently generous conception of properties, and granted the acceptability of the underlying modal logic, the theorems listed to follow from the axioms. (So say Godel, Dana Scott, Sobel, Anderson, and Adams. Who am I to disagree?) Perhaps 
one might object to the conception of properties and/or the modal logic. But one doesn't need to: the proof is demonstrably no good even if these things are accepted.

The problem -- as with virtually all ontological arguments known to me -- lies in the fact that there are parallel arguments which can be constructed, which seem no less acceptable to atheists and agnostics, but whose acceptance leads to absurd results. ${ }^{1} \mathrm{~A}$ template for constructing the parallel arguments is as follows:

Definition $1^{*}$ : $\mathrm{x}$ is God*-like iff $\mathrm{x}$ has as essential properties those and only those properties which are positive, except for $\mathrm{P}_{1}, \ldots, \mathrm{P}_{\mathrm{n}}$.

Definition 2: $\mathrm{A}$ is an essence of $\mathrm{x}$ iff for every property $\mathrm{B}, \mathrm{x}$ has $\mathrm{B}$ necessarily iff A entails B

Definition 3: $\mathrm{x}$ necessarily exists iff every essence of $\mathrm{x}$ is necessarily exemplified.

Axiom 1: If a property is positive, then its negation is not positive.

Axiom 2: Any property entailed by [= strictly implied by] a positive property is positive.

Axiom 3*: The property of being God*-like is positive.

Axiom 4: If a property is positive, then it is necessarily positive.

Axiom 5*: Necessary existence is positive, and distinct from each of $\mathrm{P}_{1}, \ldots, \mathrm{P}_{\mathrm{n}}$. Theorem 1: If a property is positive, then it is consistent [=possibly exemplified].

Corollary 1: The property of being God*-like is consistent.

Theorem 2: If something is God*-like, then the property of being God*-like is an essence of that thing.

Theorem 3: Necessarily, the property of being God*-like is exemplified.

So: for each collection of positive properties which includes necessary existence, there is a being which has exacly those positive properties, and which has no other positive properties. Even if there are only $\mathrm{N}+1$ positive properties, this gives us $2^{\mathrm{N}}$ distinct necesarily existent beings. Not good. (Perhaps $\mathrm{N}$ isn’t finite. If so, things are even worse.) 
In order to rule out the parallel argument, one might take the view that any collection of positive properties which includes necessary existence entails each of the other positive properties -- i.e. that anything which has some of the positive properties including necessary existence must have them all. This is a very strong assumption: it requires that each of the positive properties in entailed by necessary existence alone, and hence rules out the existence of any necessary beings other than one which has all the positive properties. Those who are inclined to think that numbers and/or other abstracta are necessary existents will not find this acceptable. And, in any case, there is still a problem of implementation. Suppose we add the following axiom to our original collection:

Axiom 6: Nothing which has some positive properties including necessary existence fails to be God-like

Then it seems that there is a different kind of disturbing parallel argument which can be generated. Let $* *$ be some proper subset of the positive properties which includes necessary existence; say that a property is positive** just in case it belongs to this collection. Then our template for constructing parallel arguments is as follows:

Definition $1^{* *}$ : $\mathrm{x}$ is God**-like iff $\mathrm{x}$ has as essential properties those and only those properties which are positive**.

Definition 2: A is an essence of $\mathrm{x}$ iff for every property $\mathrm{B}, \mathrm{x}$ has $\mathrm{B}$ necessarily iff $\mathrm{A}$ entails B

Definition 3: $x$ necessarily exists iff every essence of $x$ is necessarily exemplified.

Axiom $1 * *$ : If a property is positive**, then its negation is not positive**.

Axiom $2^{* *}$ : Any property entailed by [= strictly implied by $]$ a positive** property is positive**.

Axiom 3**: The property of being God**-like is positive**.

${ }^{1}$ In Oppy (1995:225), I write: "It may be possible to reinterpret the [Godelian] proof in a damaging way, though I have not been able to see how to do this”. I think that my vision has now improved a little: hence the present paper. 
Axiom $4^{* *}$ : If a property is positive**, then it is necessarily positive**.

Axiom $5^{* *}$ : Necessary existence is positive**.

Axiom6**: Nothing which has some positive** properties including necessary existence fails to be God**-like

Theorem $1^{* *}$ : If a property is positive**, then it is consistent [=possibly exemplified].

Corollary $1^{* *}$ : The property of being God**-like is consistent.

Theorem 2**: If something is God**-like, then the property of being God**-like is an essence of that thing.

Theorem 3**: Necessarily, the property of being God**-like is exemplified

Of course, defenders of the original argument will object that Axiom $6^{* *}$ is false:

nothing which is God-like is God**-like, even though anything which is God-like has some positive** properties including necessary existence. But I can’t see any reason why opponents of the original argument should be disposed to see any merit in this objection -- for it relies on the assumption that there is something which is God-like, i.e. it relies on the conclusion which the original argument was supposed to demonstrate. If there is nothing which is God-like then -- at least for all that has been said so far -- it might be that there is something which is God**-like; i.e. it might be that Axiom 6** is true, while Axiom 6 is false. So it seems that atheistic and agnostic opponents of the argument can say this: there are $2^{\mathrm{N}}$ arguments for incompatible beings, none of which provides any more reason for acceptance of its conclusion than the others. In these circumstances, it is reasonable for atheists and agnostics to reject all of the arguments; so, the original ontological argument fails.

At this point, I am inclined to draw the conclusion that the defender of the original argument is playing a losing hand. Perhaps I am wrong about this; but I do not think that I will be alone in thinking that there are no prospects for the production of a Godelian ontological argument which does not admit of these kinds of damaging parallels. (Why should atheists and agnostics think that the collection of positive 
properties is more likely to be exemplified than the collection of positive** properties? Why should they think that any such collection of properties is exemplified?)

Suppose that my conjecture is correct. Then it seems to me that we can conclude that there is more wrong with the original Godelian ontological arguments than anyone has hitherto suggested. One might, e.g., worry whether a God-like being would have the attributes traditionally ascribed to God (Adams); or perhaps one might insist that it is clear that a God-like being could not have the attributes traditionally ascribed to God (Sobel); or ... . But, if I am right, these sorts of considerations must be comparatively unimportant: the possibility of producing the kinds of parallels which I have offered shows (at least) that reasonable atheists and agnostics have good reason not to be persuaded by Godelian ontological arguments even before those other (comparatively unimportant) considerations are introduced.

Of course, there is still the question of pin-pointing the error(s) in the Godelian arguments: the production of damaging parallels may show that there is something wrong, but it does not constructively identify the error(s). I guess that reasonable atheists and agnostics are bound to say that one of the axioms is (likely) false. ${ }^{2}$ One obvious candidate is Axiom 5: granted the rest of the theoretical machinery, reasonable atheists and agnostics may (perhaps should) say that necessary existence is positive only if it is exemplified. If this is denied -- i.e. if it is granted that necessary

2 Another option -- which I categorise as a rejection of some of the theoretical machinery -would be to claim that the argument fails because no content has been given to the notion of a positive property. What are positive properties? How do we know that there are any? Etc. I follow that alternative option given in the text because it seems to require fewer controversial 
existence is positive whether or not it is exemplified, then another obvious candidate is Axiom 3: granted the rest of the theoretical machinery (including Axiom 5), atheists and agnostics may (perhaps should) say that the property of being God-like is positive only if it is exemplified. Either way, it will then be clear why the argument fails to convince: one will only think that the proof constitutes a sound argument if one is antecedently convinced of the truth of its conclusion. (Moreover -- though I shan't try to argue for this claim here -- it seems to me that once it is conceded that the argument does not constitute a proof which ought to persuade atheists and agnostics of the truth of its conclusion, then it is conceded that the argument is utterly worthless. It has all and only the virtues of this (classical) argument: "Either 2+2=5 or God exists; It is not the case that 2+2=5; Therefore God exists” -- an argument which all theists should think is sound -- at least granted the stipulation that the logical connectives are all to be interpretted classically -- but which has no other virtues whatsoever. ${ }^{3}$ )

Research School for the Social Sciences

Australian National University

Canberra ACT 0200, Australia

oppy@coombs.anu.edu.au

References

commitments: even if some content can be given to the notion of a positive property, the Godelian ontological arguments will still fail. 
Adams, R. M. 1995. Introductory Note to *1970. In Collected Works Volume III: Unpublished essays and lectures, K. Godel (editor-in-chief Solomon Feferman), 388-402. New York: Oxford University Press.

Anderson, C. A. 1990. Some emendations on Godel's ontological proof. Faith and Philosophy 7: 291-303.

Oppy, G. 1995. Ontological arguments and belief in God. New York: Cambridge University Press.

Sobel, J. H. 1987. Godel's Ontological Proof. In On being and saying: essays for Richard Cartwright, ed. J. J. Thomson, 241-261. Cambridge, Mass.: MIT Press.

${ }^{3}$ For argument in support of this contention, see Oppy (1995), in particular Chapter 12. 